\title{
Neoadjuvant chemotherapy with carboplatin and docetaxel in advanced ovarian cancer - a prospective multicenter phase II trial (PRIMOVAR)
}

\author{
MARTIN PÖLCHER $^{1}$, SVEN MAHNER $^{2}$, OLAF ORTMANN $^{3}$, JÖRN HILFRICH $^{4}$, KLAUS DIEDRICH $^{5}$, \\ GEORG-PETER BREITBACH ${ }^{6}$, CORNELIA HÖSS ${ }^{7}$, CLAUDIA LEUTNER $^{8}$, MICHAEL BRAUN $^{1}$, \\ VOLKER MÖBUS ${ }^{9}$, INA KARBE $^{10}$, PATRICK STIMMLER ${ }^{3}$, CHRISTIAN RUDLOWSKI $^{1}$, \\ JÖRG SCHWARZ ${ }^{2}$ and WALTHER KUHN ${ }^{1}$
}

\begin{abstract}
${ }^{1}$ Department of Gynecology and Obstetrics, Center for Integrated Oncology, University of Bonn; ${ }^{2}$ Department of Gynecology, University Medical Center Hamburg-Eppendorf; ${ }^{3}$ Department of Gynecology and Obstetrics, University of Regensburg; ${ }^{4}$ Diakoniekrankenhaus Henriettenstiftung, Hannover; ${ }^{5}$ Department of Gynecology and Obstetrics, University of Lübeck; ${ }^{6}$ Städtisches Klinikum Neunkirchen; ${ }^{7}$ Kreisklinik Ebersberg; ${ }^{8}$ Department of Radiology, University of Bonn;

${ }^{9}$ Städtisches Klinikum Frankfurt; ${ }^{10}$ Department of Gynecology and Obstetrics, University of Halle, Germany
\end{abstract}

Received March 30, 2009; Accepted April 29, 2009

DOI: 10.3892/or_00000479

\begin{abstract}
Early response criteria and surgical outcome were evaluated in patients with advanced epithelial ovarian cancer treated with neoadjuvant chemotherapy. Patients with FIGO stage IIIC or IV ovarian cancer and an ascites volume of $\geq 500 \mathrm{ml}$ were randomly assigned to receive preoperatively 3 (A1) or 2 (A2) of 6 cycles of carboplatin and docetaxel intravenously. Response was monitored by measuring target lesions, ascites volumes and serum CA 125 levels. The primary outcome measure was the preoperative reduction of ascites volume. Secondary outcome measures were the evaluation of residual tumor and perioperative morbidity and mortality. Eighty-three patients underwent cytoreductive surgery, 40 after 3 cycles and 43 patients after 2 cycles of neoadjuvant chemotherapy. 'Optimal debulking' $(\leq 1 \mathrm{~cm})$ was achieved in 30 (A1) and 32 patients (A2). Eight (A1) and 6 patients (A2) had a persistent ascites volume $\geq 500 \mathrm{ml}$. A decrease of the CA 125 level from baseline of less than $50 \%$ was observed in 7 (A1) and 9 patients (A2). Computed tomography scan results showed progressive disease in 6 patients (3 A1; $3 \mathrm{~A} 2)$. Any amount of residual disease after cytoreductive surgery, persistent ascites, and a less pronounced decrease of CA 125 were associated with poor
\end{abstract}

Correspondence to: Dr Martin Pölcher, Department of Gynecology and Obstetrics, Center for Integrated Oncology, University of Bonn, Sigmund-Freud-Str. 25, 53105 Bonn, Germany

E-mail: martin.poelcher@ukb.uni-bonn.de

Key words: ovarian cancer, neoadjuvant chemotherapy, surgery, docetaxel, CA 125, ascites progression-free survival rates. In conclusion, ascites volume reduction and CA 125 decline appear to be appropriate response criteria. A treatment schedule with two preoperative cycles is a reasonable option for neoad-juvant chemotherapy in advanced ovarian cancer. High surgical standards are mandatory, even after neoadjuvant chemotherapy.

\section{Introduction}

Epithelial ovarian cancer (EOC) is the sixth most common cancer in women, and accounts for more than 100,000 deaths a year worldwide (1). Patients with International Federation of Gynecology and Obstetrics (FIGO) stage IIIC and IV disease have an unfavorable prognosis with 5-year survival rates from 19 to $33 \%$ depending on resectability and response to chemotherapy (2).

Apart from the FIGO stage, the postoperative residual tumor diameter is the most important prognostic factor for survival. Despite high surgical effort, 'optimal cytoreduction' $(\leq 1 \mathrm{~cm})$ is only achieved in $70-80 \%$ even in the most experienced centers; $20-30 \%$ of the patients do not benefit quo ad vitam from an extensive operation $(3,4)$.

The standard therapy of advanced ovarian cancer is cytoreductive surgery followed by platinum and taxane-based chemotherapy. The presence of malignant ascites is an established independent prognostic factor in retrospective analyses (2). Large volume of ascites $(>500 \mathrm{ml})$ is consistent with diffuse peritoneal carcinomatosis and correlates with a low probability of achieving optimal tumor resection. Neoadjuvant chemotherapy could be an option to achieve higher resection rates in this group of patients $(2,5,6)$. A prospective non-randomized phase II study of patients with FIGO IIIC EOC and more than $500 \mathrm{ml}$ ascites showed a significant improvement in survival rates after neoadjuvant chemotherapy compared to the standard approach (42 vs. 23 months) (6). 
Although numerous other non-randomized studies exploring the role of neoadjuvant chemotherapy have been published, the true impact of preoperative treatment has not been finally established $(7,8)$. The majority of EOC patients studied so far in the setting of neoadjuvant chemotherapy were given three or four cycles preoperatively (9). An increased number of cycles given preoperatively could promote the formation of resistant cell clones in large tumors (10); by the same token microscopic or small residual disease might not be eliminated completely by only a few postoperative cycles (11). In a recently performed meta-analysis on 22 cohorts of patients with FIGO stage III or IV the authors concluded that within the range of three to six median cycles prior to cytoreductive surgery, each additional cycle of chemotherapy was associated with an incremental decrease in median cohort survival time of 4.1 months (12).

The purpose of this study was to determine a suitable regimen for a planned phase III study (neoadjuvant chemotherapy vs. standard therapy) by evaluating efficacy, tolerability and surgical outcome in patients treated with preoperative chemotherapy with carboplatin/docetaxel. Furthermore we explored if biological and anatomical markers could provide an indication of response prior to cytoreductive.

\section{Material and methods}

Eligibility. This study included patients with histologically confirmed FIGO stage IIIC and IV epithelial ovarian cancer and an ascites volume of $500 \mathrm{ml}$ or more. The ascites volume was measured after draining during initial surgery or estimated by imaging in cases of biopsy-proven cancer. The main exclusion criteria were mucinous cell type, debulking procedures during initial surgery, and signs of bowel obstruction. All patients provided informed consent and this investigation followed Good Clinical Practice guidelines. The protocol was approved by the ethics committee at each participating center. The study was monitored by an independent clinical research organization in accordance with a predefined schedule.

Treatment plan. Patients were randomly assigned to receive either three (A1) or two (A2) of six cycles of intravenous carboplatin (AUC 5) and $75 \mathrm{mg} / \mathrm{m}^{2}$ of docetaxel every 3 weeks before cytoreductive surgery. If disease progression occurred, further treatment was initiated at the discretion of the investigator, and deviation from the treatment plan was not considered to be a violation of the protocol.

Safety. Adverse events and toxicities were graded by study investigators in accordance with the National Cancer Institute (NCI) Common Toxicity Criteria (CTC) (version 2/1998) and were recorded continuously.

Clinical assessments. At baseline, chest X-rays, CT scans or MRI and vaginal ultrasounds were performed to determine target lesions and to confirm a large volume of ascites. Imaging was repeated after two cycles (entire group) and three cycles (arm A1). Target lesions were measured in accordance with standard World Health Organization (WHO) response criteria (13). Serum CA 125 levels were measured before therapy and after each cycle of chemotherapy. CA 125 levels before cytoreductive surgery were compared to baseline, and a decrease of $50 \%$ or more was considered to be an indicator of response. Follow-up was carried out for each patient every three months.

Surgery. Cytoreductive surgery had to be performed within four weeks of the last scheduled chemotherapy cycle. During the operation, parallel to the standard approach all surgical staging procedures should be performed as well as necessary intestinal resections and extended upper abdominal surgery in order to remove all tumor manifestations as far as possible (14). 'Optimal debulking' was defined as residual tumor of $\leq 1 \mathrm{~cm}$ in diameter.

Statistical design and methods. The study was designed as a two-arm randomized selection study comparing two different regimens of neoadjuvant chemotherapy in which each arm followed a single-stage design. In a previous study the ascites rate, i.e., to reduce the preoperative ascites volume to less than $500 \mathrm{ml}$ was reported to be $70 \%$ (6). To consider the preoperative chemotherapy as useful a success rate of $70 \%$ or more should be achieved (primary study end point). If it was less than $50 \%$ the procedure would be unacceptable. With a $95 \%$ chance (alpha $=5 \%$ ) of rejecting the procedure if the ascites volume reduction rate was only $50 \%$, and with a $80 \%$ chance of concluding the procedure as worthwhile if it is $70 \%$ or more, 37 patients have to be enrolled (15). If both arms would pass the success criterion, arm A2 will be preferred if the relative success rate is not worse than 5\% compared to arm A1. Anticipating a drop-out rate of $10 \%$ a total of 41 patients per arm was planned to be enrolled into the study. Secondary endpoints were the evaluation of residual disease after surgery and perioperative morbidity and mortality.

Differences between demographic data and outcome variables were tested with Fisher's exact test or $\chi^{2}$-test where appropriate. P-values are two-sided and significant at $\mathrm{p}<0.05$. The Cox proportional hazards model was used to identify the independent prognostic factors as well as to estimate their effects on PFS and OS adjusted for covariates. Estimates of the cumulative proportions of survival were based on the Kaplan-Meier method (software SAS 9.1.3, SAS Institute Inc. Cary, NC, USA).

\section{Results}

Recruitment and treatment received. Ninety-three patients were enrolled at 9 centers (1-35 patients) from February 2003 through January 2008. Eighty-eight received at least one cycle of carboplatin/docetaxel and were assessed for the Intentionto-treat population. Four patients did not receive study medication, one patient died before treatment (Fig. 1).

Both treatment groups were well balanced for known prognostic factors and patient characteristics. There were no significant differences in the arms (Table I). No major differences in patient characteristics and results were noted between the various centers.

Adverse effects and toxicity. A total of 467 cycles of chemotherapy were administered. The most frequent grade 3 or 4 
93 Registration and randomization ${ }^{\circ}$

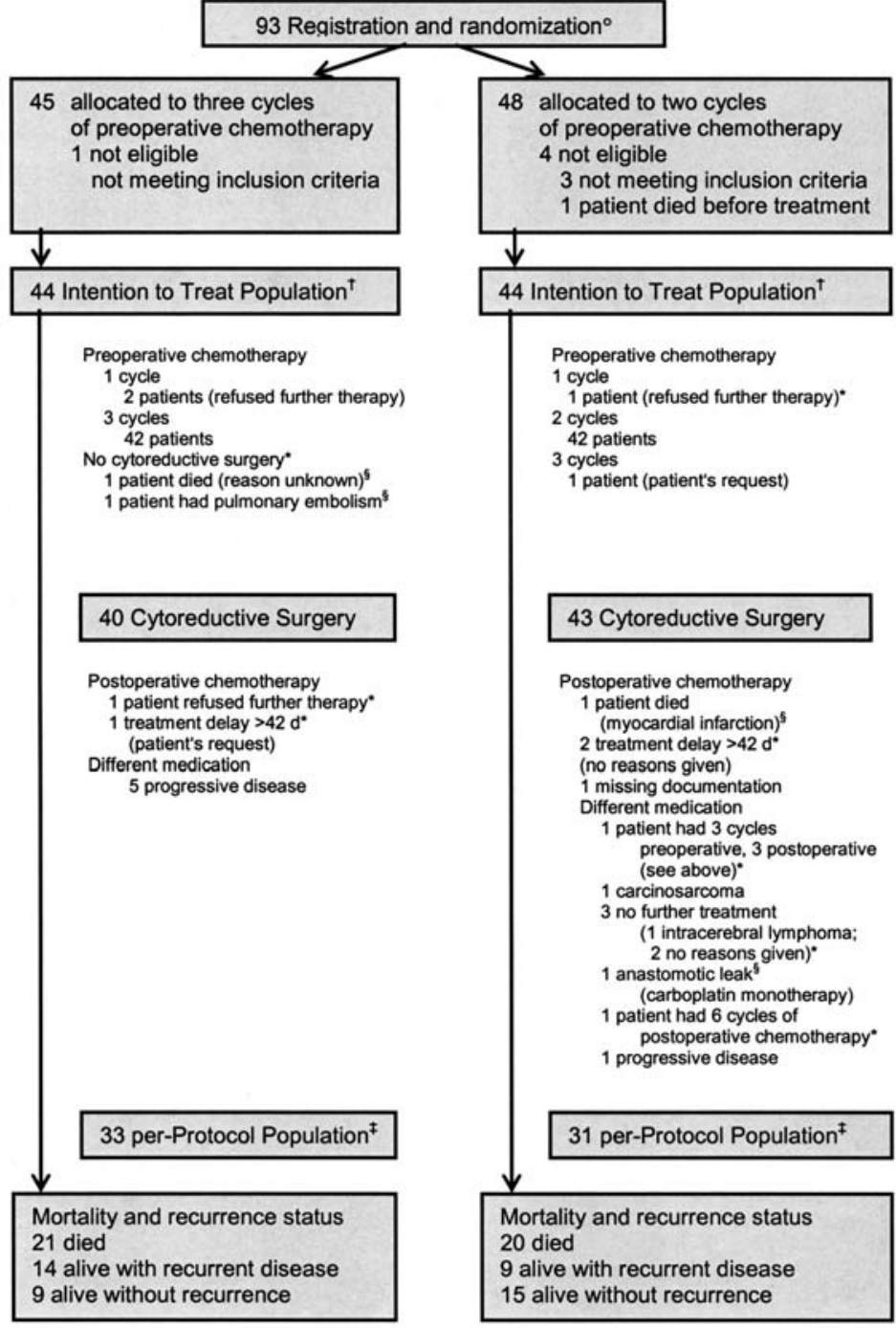

- central randomization via fax, computer generated randomization lists for each participating center (Software PROC PLAN, SAS), allocation concealed.

$t$ at least one cycle of preoperative study medication.

Protocol violation.

$\S$ Serious Adverse Event, Serious Unexpected Adverse Event.

$\ddagger$ Therapy ended as planned in protocol (therapy adjustments in cases of progressive disease was not a violation of protocol).

Figure 1. CONSORT Diagram (35).

toxicity was leucopenia, which occurred in $65(74 \%)$ of the patients. Treatment delays before and after cytoreductive surgery are shown in Table II. The most frequently cited reasons for treatment delay were organizational and not medical. Relaparotomy was done in five cases $(6 \%)$ due to complications (secondary bleeding, $\mathrm{n}=4$; anastomotic leak, $\mathrm{n}=1$ ). There were no deaths within 30 days following cytoreductive surgery.

Results of cytoreductive surgery. After receiving neoadjuvant therapy 83 patients were operated. 'Optimal debulking' was achieved in 62 patients $(75 \%), 31(38 \%)$ had no macroscopic residual disease (Table II).

Preoperative response evaluation. At the time of cytoreductive surgery, 29 and 36 of 79 eligible patients had less than $500 \mathrm{ml}$ of ascites (corresponding to $78 \%$ in A1 and $86 \%$ in A2). The success criterion was fulfilled in both arms. Fourteen patients
(18\%) had more than $500 \mathrm{ml}$ of ascites (8 in A1 and 6 in A2) (Table III, Fig. 2).

All patients had elevated CA 125 serum levels at the time of randomization and only one patient had a baseline CA 125 level $<70 \mathrm{U} / \mathrm{ml}$, which is twice the upper limit of normal. All values decreased after the initial two cycles of carboplatin/ docetaxel.

In 58 patients $(66 \%)$, the extent of the disease was monitored using computed tomography. Forty-nine CT scans were evaluated after the second preoperative cycle $(56 \%$ of the total group), $26 \mathrm{CT}$ scans after the third cycle (59\% of patients treated in A1). Findings from all three investigations were available for 21 patients (47\%). The reasons for missing data were omission, changes in the imaging modality or methodical difficulties such as non-measurable disease.

Survival. The median follow-up time was 15.6 months (range 4-53 months). A total of 64 patients $(73 \%)$ showed a 
Table I. Patient demographic and clinical characteristics.

\begin{tabular}{|c|c|c|c|}
\hline & Total & Arm A1 $(3+3$ cycles $)$ & Arm A2 ( $2+4$ cycles $)$ \\
\hline Number of patients & 88 & 44 & 44 \\
\hline \multicolumn{4}{|l|}{ Age } \\
\hline Median & 64 & 65 & 63 \\
\hline Range & $39-80$ & $43-79$ & $39-80$ \\
\hline \multicolumn{4}{|c|}{ ECOG performance status-no. (\%) } \\
\hline 0 & $29(33 \%)$ & $20(45 \%)$ & $9(20 \%)$ \\
\hline 1 & $39(44 \%)$ & $15(34 \%)$ & $24(55 \%)$ \\
\hline 2 & $8(9 \%)$ & $5(11 \%)$ & $3(7 \%)$ \\
\hline Not specified $(\leq 2)$ & $12(14 \%)$ & $4(9 \%)$ & $8(18 \%)$ \\
\hline \multicolumn{4}{|l|}{ CA $125(\mathrm{U} / \mathrm{ml})$} \\
\hline Median & 1447 & 931 & 2033 \\
\hline Range & $43-90400$ & $43-69044$ & $197-90400$ \\
\hline \multicolumn{4}{|l|}{ Ascites (ml) } \\
\hline Median & 1500 & 1300 & 1500 \\
\hline Range & $(500-6500)$ & $(500-5000)$ & $(500-6500)$ \\
\hline \multicolumn{4}{|c|}{ Staging procedure-no. $(\%)$} \\
\hline Laparoscopy & $68(77 \%)$ & $33(75 \%)$ & $35(80 \%)$ \\
\hline Laparotomy & $16(18 \%)$ & $9(20 \%)$ & $7(16 \%)$ \\
\hline Other ${ }^{\mathrm{a}}$ & $4(5 \%)$ & $2(5 \%)$ & $2(5 \%)$ \\
\hline \multicolumn{4}{|c|}{ Histological type-no. (\%) } \\
\hline Serous carcinoma & $81(92 \%)$ & $39(89 \%)$ & $42(95 \%)$ \\
\hline Adenocarcinoma, & & & \\
\hline Unspecified & $6(7 \%)$ & $4(9 \%)$ & $2(5 \%)$ \\
\hline Other $^{\mathrm{b}}$ & $1(1 \%)$ & $1(2 \%)$ & - \\
\hline \multicolumn{4}{|c|}{ Histological grade-no. (\%) } \\
\hline Otherb & $1(1 \%)$ & $1(2 \%)$ & 0 \\
\hline G 2 & $24(27 \%)$ & $13(30 \%)$ & $11(25 \%)$ \\
\hline G 3 & $63(72 \%)$ & $30(68 \%)$ & $33(75 \%)$ \\
\hline \multicolumn{4}{|l|}{ FIGO stage-no. (\%) } \\
\hline IIIC & $64(73 \%)$ & $32(73 \%)$ & $32(73 \%)$ \\
\hline IV & $24(27 \%)$ & $12(27 \%)$ & $12(27 \%)$ \\
\hline
\end{tabular}

ECOG, Eastern Cooperative Oncology Group; FIGO, Federation of Obstetrics and Gynecology. ${ }^{\mathrm{a} C T}$ scan guided biopsy (3), biopsy via colonoscopy (1) and ${ }^{\mathrm{b}}$ carcinosarcoma.

progression or recurrence of the disease within the observation period. Forty-one patients $(47 \%)$ had died by the end of the observation period, nevertheless OS rates are listed.

Between both treatment arms there were no significant differences in the PFS rates [A1 median 12.2 months, 95\% confidence interval (CI) $10.8-14.5$ vs. A2 12.5 months, $95 \%$ CI 9.4-17.0; $\mathrm{p}=0.77]$ and OS rates. (Median 24.1 months, 95\% CI 17.9-33.7 vs. 28.4 months, 95\% CI 16.0-36.6; $\mathrm{p}=0.87$ ). As there were no significant discrepancies in the secondary study aims, an analysis among the entire group was carried out to explore the prognostic value of the proposed response criteria (Table IV).

\section{Discussion}

Neoadjuvant chemotherapy in solid tumors has been generally considered beneficial, mainly for two reasons: down-staging can improve surgical outcome, and the response to treatment can reflect chemosensitivity and might guide further therapy. Both could be advantageous in the treatment of ovarian cancer patients: owing to the paucity of symptoms and their insidious onset, most patients are diagnosed with large intra-abdominal tumor spread. Patients without macroscopic or with only minimal disease left ('optimal debulking' $\leq 1 \mathrm{~cm}$ in diameter) experience a superior outcome compared to patients who undergo 'suboptimal' cytoreductive surgery. The latter group sustain the morbidity of a cytoreductive attempt without an associated survival benefit. In both, highly chemosensitive and chemoresistant disease is observed. A number of intrinsic or acquired resistance mechanisms hamper the effectiveness of chemotherapy resulting in early recurrence after initial treatment in up to $20 \%$ of the patients (2). These unfavorable conditions continue to present a clinical problem.

In this study the concept of neoadjuvant chemotherapy should be appraised. Large volume ascites was chosen as an inclusion criterion to select a subgroup of ovarian cancer 
Table II. Results of cytoreductive surgery.

\begin{tabular}{|c|c|c|c|}
\hline & Arm A1 ( $3+3$ cycles $)$ & Arm A2 ( $2+4$ cycles $)$ & p-value \\
\hline Number of patients & 40 & 43 & \\
\hline $\begin{array}{l}\text { Tumor resection rate-no. }(\%) \\
\text { Optimal debulking }(\leq 1 \mathrm{~cm}) \\
\text { no gross residual disease } \\
\text { macroscopic } \leq 1 \mathrm{~cm} \\
\text { Suboptimal debulking }(>1 \mathrm{~cm}) \\
\text { Not known }\end{array}$ & $\begin{array}{l}30(75 \%) \\
12(30 \%) \\
18(45 \%) \\
8(20 \%) \\
2(5 \%)\end{array}$ & $\begin{array}{l}32(74 \%) \\
19(44 \%) \\
13(30 \%) \\
11(26 \%)\end{array}$ & NS \\
\hline $\begin{array}{l}\text { Operation time }(\mathrm{h}) \\
\text { Mean } \\
\text { Median } \\
\text { Range }\end{array}$ & $\begin{array}{c}5.55 \pm 1.93 \\
6 \\
2-9\end{array}$ & $\begin{array}{c}5.52 \pm 1.88 \\
6 \\
1-9\end{array}$ & NS \\
\hline $\begin{array}{l}\text { Pelvic and para-aortic lymph node dissection } \\
\text { No. }(\%)\end{array}$ & $23(58 \%)$ & $23(53 \%)$ & \\
\hline $\begin{array}{l}\text { Infragastric omentectomy } \\
\text { No. }(\%)\end{array}$ & $36(90 \%)$ & $40(94 \%)$ & \\
\hline $\begin{array}{l}\text { Upper abdominal surgery procedures }{ }^{\mathrm{a}} \\
\text { no. }(\%)\end{array}$ & $13(33 \%)$ & $12(28 \%)$ & NS \\
\hline $\begin{array}{l}\text { Bowel resection }{ }^{\mathrm{b}} \\
\text { No. }(\%)\end{array}$ & $13(33 \%)$ & $17(40 \%)$ & NS \\
\hline $\begin{array}{l}\text { Time to cytoreductive surgery after the last } \\
\text { scheduled chemotherapy cycle }-(\mathrm{d}) \\
\text { Within } 28 \mathrm{~d} \\
29-42 \mathrm{~d} \\
>42 \mathrm{~d}^{\mathrm{c}}\end{array}$ & $\begin{array}{c}31(78 \%) \\
8(20 \%) \\
1(3 \%)\end{array}$ & $\begin{array}{c}32(74 \%) \\
10(23 \%) \\
1(2 \%)\end{array}$ & NS \\
\hline $\begin{array}{l}\text { Time until first cycle of chemotherapy } \\
\text { after cytoreductive surgery-(d) } \\
\text { Within } 28 \text { d } \\
29-42 \text { d } \\
>42 d^{\text {c }}\end{array}$ & $\begin{array}{c}33(83 \%) \\
2(5 \%) \\
1(3 \%)\end{array}$ & $\begin{array}{c}35(81 \%) \\
2(5 \%) \\
1(3 \%)\end{array}$ & NS \\
\hline
\end{tabular}

${ }^{\text {aS }}$ urgical procedures such as diaphragm resection, splenectomy or cholecystectomy; ${ }^{\mathrm{b}}$ large or small bowel resection; ${ }^{\mathrm{c}}$ protocol violation.

patients with unfavourable outcome. Patients who present with ascites volume $\geq 500 \mathrm{ml}$ are more likely to have peritoneal carcinomatosis and bulky upper abdominal disease cephalad to the greater omentum (16). Despite paracentesis, ascites rapidly recreates without efficacious therapy. In a previous examination $70 \%$ of patients had no or only a small volume of ascites after 3 cycles of preoperative chemotherapy (6). The ascites volume at the moment of cytoreductive surgery is analyzed as a response criterion in this study. The proposed response rate of $70 \%$ was achieved in both treatment arms, there was no significant discrepancy. The primary aim of the study was therefore achieved. A comparison of the secondary endpoints, i.e., residual tumor and perioperative morbidity and mortality, also revealed no significant differences, suggesting that 2 cycles are not inferior to 3 . Taking a potential induction of chemoresistance into account and considering an effective treatment of microscopic or small residual disease, the regimen with 2 preoperative cycles and 4 cycles after cytoreductive surgery is preferable for follow-up studies-as determined before hand.
Retrospective studies show high resection rates after neoadjuvant chemotherapy (17). These results were confirmed in this investigation, in which 'optimal debulking', i.e., a residual tumor diameter $\leq 1 \mathrm{~cm}$, was achieved in $75 \%$ of these selected patients with large volume ascites. Mean operation time was $5.5 \mathrm{~h}$, which indicates the necessity of complex surgical procedures.

However, the resection rates achieved in upfront cytoreductive surgery in the same patient group are not clear and the accuracy of the inclusion criterion of $\geq 500 \mathrm{ml}$ ascites as a predictor of residual disease after cytoreductive surgery has not been validated prospectively. Several investigators have attempted to describe preoperative predictors of surgical outcome. Independent radiologic predictors of suboptimal cytoreduction failed to show sufficient accuracy when applied to different cohorts of patients (18). Baseline CA 125 levels in this study were considerably increased (median $1447 \mathrm{U} / \mathrm{ml}$ ), but preoperative serum CA 125 levels have failed to be precise enough to predict cytoreductive outcomes at least with a cut-off-level of 400 or $500 \mathrm{U} / \mathrm{ml}$ (19-21). Alternative approaches 


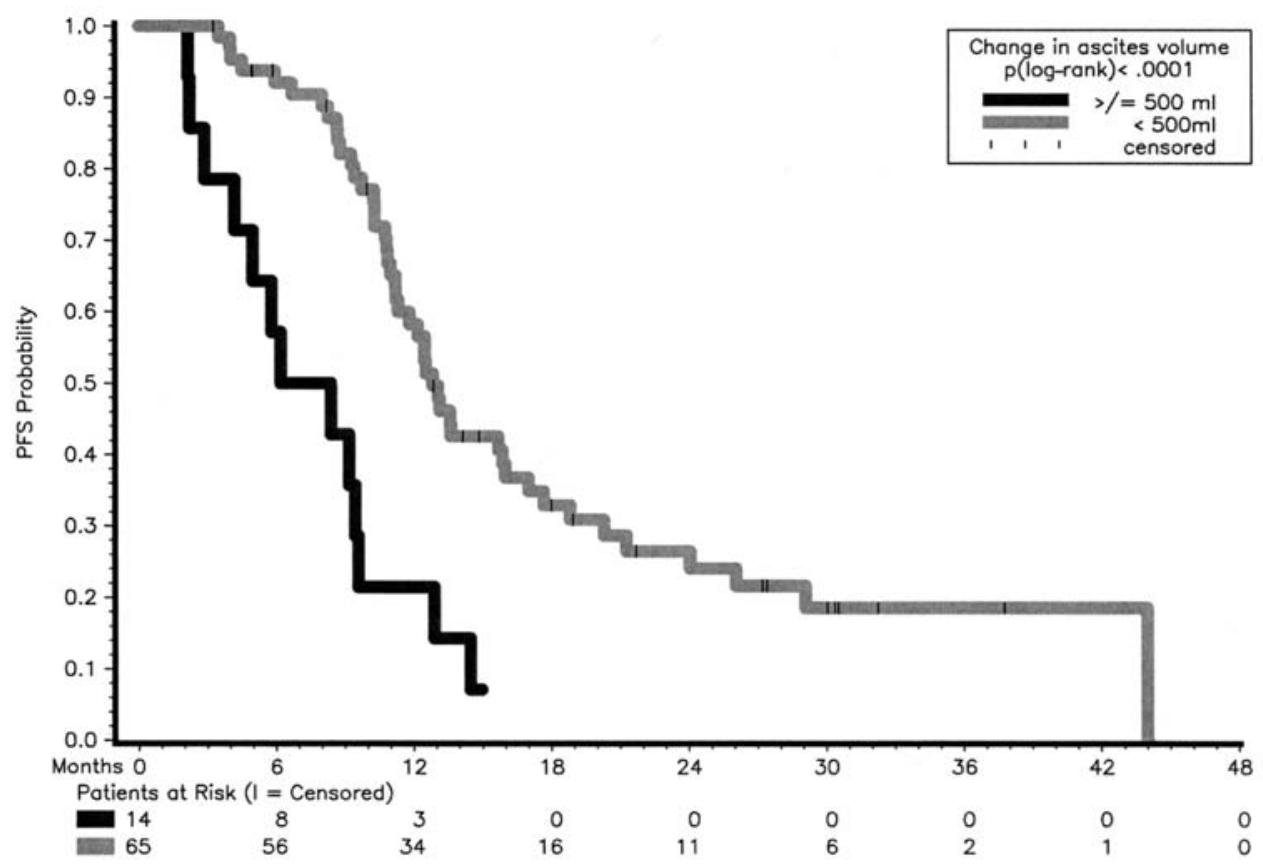

Figure 2. Kaplan-Meier estimate of progression-free survival (PFS) (entire group) by change in ascites volume ( $<500 \mathrm{ml} \mathrm{vs}$. $\geq 500 \mathrm{ml}$ ascites at the time of cytoreductive surgery).

Table III. Response evaluation prior to cytoreductive surgery (Intention-to-Treat-Population): proportion of 'non-responder'.

\begin{tabular}{|l|c|c|c|}
\hline & $\begin{array}{c}\text { Persisting large volume } \\
\text { ascites }>500 \mathrm{ml}\end{array}$ & $\begin{array}{c}\text { CA 125 serum level: } \\
\text { decrease }<50 \%\end{array}$ & $\begin{array}{c}\text { Target lesion CT scan: } \\
\text { WHO criteria }\end{array}$ \\
\hline $\begin{array}{l}\text { Arm A 1 } \\
\text { (after 3 cycles } \\
\text { preoperative } \\
\text { chemotherapy) }\end{array}$ & not available: 7 & & \\
\hline $\begin{array}{l}\text { Arm A 2 } \\
\text { (after 2 cycles } \\
\text { preoperative } \\
\text { chemotherapy) }\end{array}$ & not available: 2 & & \\
\hline
\end{tabular}

$\mathrm{CR}$, complete response; PR, partial response; $\mathrm{SD}$, stable disease; $\mathrm{PD}$, progressive disease.

including the use of laparoscopy to determine the chances of optimal cytoreduction are promising but also underlie the subjective evaluation of the surgeon and, to date, are difficult to apply to different institutions (22).

Moreover, the threshold $\leq 1 \mathrm{~cm}$ was adopted from current standard treatment and represents the defining outcome measure of surgery which significantly influence survival rates (3). It has not been proven that it has the same prognostic impact after neoadjuvant chemotherapy: in this study, patients with any amount of macroscopic residual tumor had poor PFS and OS rates compared to patients without gross residual disease. It may be assumed that only patients without residual disease left after neoadjuvant chemotherapy and cytoreductive surgery will benefit from this approach. 'Optimal debulking' after neoadjuvant chemotherapy should be defined as no gross residual disease.

Response to chemotherapy is considered an important prognostic factor for survival and tumor shrinkage is a common 
Table IV. Univariate and mulitvariate logistic regression modeling analyzing parameters associated with progression-free survival (PFS) and overall survival (OS).

\begin{tabular}{|c|c|c|c|c|c|c|c|c|c|c|c|c|}
\hline & \multicolumn{6}{|c|}{ Univariate } & \multicolumn{6}{|c|}{ Multivariate } \\
\hline & \multicolumn{3}{|c|}{ PFS } & \multicolumn{3}{|c|}{ OS } & \multicolumn{3}{|c|}{ PFS } & \multicolumn{3}{|c|}{ OS } \\
\hline & $\mathrm{p}$ & HR & $\mathrm{HR} 95 \% \mathrm{Cl}$ & $\mathrm{p}$ & HR & $\mathrm{HR} 95 \% \mathrm{Cl}$ & $\mathrm{p}$ & $\mathrm{HR}$ & $\mathrm{HR} 95 \% \mathrm{Cl}$ & $\mathrm{p}$ & HR & $\mathrm{HR} 95 \% \mathrm{Cl}$ \\
\hline FIGO stage IIIC vs. IV & 0.13 & 0.66 & $0.39-1.13$ & 0.02 & 0.47 & $0.25-0.88$ & 0.21 & 0.69 & $0.38-1.24$ & 0.09 & 0.53 & $0.56-1.11$ \\
\hline \multicolumn{13}{|l|}{ Decrease of CA 125 level } \\
\hline$\geq 50 \%$ vs. $<50 \%$ & 0.02 & 0.71 & $0.52-0.95$ & 0.04 & 0.68 & $0.46-0.99$ & 0.31 & 0.85 & $0.62-1.16$ & 0.66 & 0.92 & $0.62-1.35$ \\
\hline \multicolumn{13}{|l|}{ Ascites volume reduction } \\
\hline$<500 \mathrm{ml}$ vs. $\geq 500 \mathrm{ml}$ & $<0.001$ & 0.29 & $0.15-0.55$ & 0.07 & 0.50 & $0.24-1.07$ & $<0.001$ & 0.21 & $0.10-0.42$ & 0.37 & 0.69 & $0.30-1.58$ \\
\hline $\begin{array}{l}\text { No residual tumor vs. } \\
\text { residual tumor }\end{array}$ & $<0.001$ & 0.37 & $0.21-0.66$ & $<0.001$ & 0.30 & $0.14-0.65$ & $<0.001$ & 0.33 & $0.17-0.64$ & $<0.001$ & 0.33 & $0.14-0.75$ \\
\hline
\end{tabular}

p, p-value; HR, hazard ratio; HR 95\% CI, hazard ratio 95\% confidence interval; PFS, progression-free survival; OS, overall survival.

endpoint used in screening new cytotoxic agents in metastatic cancer. Therefore an exploratory joint analysis was performed. Patients in whom more than $500 \mathrm{ml}$ of ascites was measured at the time of cytoreductive surgery had decreased PFS rates compared to patients who had no or less than $500 \mathrm{ml}$ of ascites.

The decline of the tumor marker CA 125 to half of its initial value before cytoreductive surgery was applied to define responders and non-responders and provides a significant difference in PFS in patients after neoadjuvant therapy. A rapid fall in the tumor marker could indicate a chemosensitive tumor and the percentage of decline may be a factor in the success of the therapy. These findings are supported by retrospectively collected data (23). In cases of recurrent disease this parameter is an established criterion of response (24).

Classification in the categories PR, SD and PD revealed no significant discrepancies as regards PFS. Target lesions could not always be determined or followed up in the next scan and therefore not all of the patients could be considered in the analysis. Furthermore cystic-solid adnexal masses were frequently described as target lesions, the reduction of which was possibly not equivalent to that of solid lesions. After excluding cystic lesions, an application of the Response Evaluation Criteria in Solid Tumors (RECIST) (25) instead of the WHO criteria achieved the same result (data not shown). A preoperative response evaluation by CT scan therefore seems to be less suitable. Established response criteria (Gynecologic Cancer Intergroup GCIG, RECIST), however, provide for confirmatory examinations at intervals of 28 days, which could not be applied in this protocol due to the surgical intervention.

Neoadjuvant chemotherapy might be an opportunity to guide therapy, i.e., to switch to a non-cross-resistant or less toxic regimen after surgery. According to these criteria about every fifth patient can be considered to be a non-responding candidate. However, no definitive conclusion can be drawn on the basis of these exploratory findings. The prognostic markers for the PFS were not confirmed in the OS analyses and the most important poor prognostic factor was the presence of residual tumor after cytoreductive surgery. This evaluation is further limited by the relatively small number of patients and the short follow-up period. Though, in addition to the clinical markers, comparing pre- and post-treatment samples and analyzing changes in signaling pathways might be an interesting tool for the response evaluation and could help to select new treatment drugs for phase III studies.

The combination docetaxel and carboplatin (DC) was chosen for study medication. Compared to standard therapy with paclitaxel/carboplatin (PC), DC produced fewer unfavorable side-effects as regards neurotoxicity, and therapy was terminated less frequently (26). PFS and OS rates showed no differences $(27,28)$.

In this study, a higher hematological toxicity associated with DC compared to PC did not have substantial influence on adherence to the treatment schedule. There were no studies of complications relating to immune suppression caused by chemotherapy, such as increased infections or inadequate wound healing. After initial treatment without pathological findings, one patient was found dead at home before the planned cytoreductive surgery could take place; the cause of death remained unclear. Comparable with standard therapy trials, $73 \%$ of the patients completed the treatment in accordance with the protocol (29). Despite the complexity of the surgical procedures, perioperative morbidity was low and the therapy generally feasible and safe.

Studies evaluating neoadjuvant chemotherapy in advanced ovarian cancer have produced mixed results and have set off a highly controversial discussion $(6,8,12,30-33)$. First results of a phase III study with 704 patients enrolled were reported by Vergote at the 12th Biennial Meeting of the International Gynecological Cancer Society: a treatment schedule with 3 cycles carboplatin/paclitaxel preoperatively showed that neoadjuvant chemotherapy produced similar OS and PFS outcomes compared to standard primary debulking and was considered as the preferred treatment due to lower morbidity data (34). 
As far as we are aware this is the first prospective multicenter phase 2 study in neoadjuvant chemotherapy. The results of this study suggest that preoperative response can be evaluated after 2 cycles and further investigations should be performed to individualize therapy for poorly responding patients. However, chemotherapy could not compensate for surgery, the most important goal is the resection of any macroscopic tumor. Therefore, high surgical standards are an essential condition for the treatment of advanced ovarian cancer, even after neoadjuvant chemotherapy. Conceivable efforts to improve surgical outcome are mandatory.

\section{Acknowledgements}

All statistical analyses were made by Michael Scholz, Trium Analysis Online $\mathrm{GmbH}$; his support is appreciated. The help of SKM Oncology/i3 research is acknowledged for the extensive data management required during this study. We also thank all clinicians who entered their patients in this PRIMOVAR-1 clinical trial.

\section{References}

1. Parkin DM, Bray FI and Devesa SS: Cancer burden in the year 2000. The global picture. Eur J Cancer 37 (Suppl 8): S4-S66, 2001.

2. Heintz AP, Odicino F, Maisonneuve P, Quinn MA, Benedet JL, Creasman WT, Ngan HY, Pecorelli S and Beller U: Carcinoma of the ovary. FIGO 6th Annual Report on the Results of Treatment in Gynecological Cancer. Int J Gynaecol Obstet 95 (Suppl 10): S161-S192, 2006.

3. Bristow RE, Tomacruz RS, Armstrong DK, Trimble EL and Montz FJ: Survival effect of maximal cytoreductive surgery for advanced ovarian carcinoma during the platinum era: a metaanalysis. J Clin Oncol 20: 1248-1259, 2002.

4. Hoskins WJ, McGuire WP, Brady MF, Homesley HD, Creasman WT, Berman M, Ball H and Berek JS: The effect of diameter of largest residual disease on survival after primary cytoreductive surgery in patients with suboptimal residual epithelial ovarian carcinoma. Am J Obstet Gynecol 170: 974-980, 1994.

5. Aletti GD, Dowdy SC, Gostout BS, Jones MB, Stanhope CR, Wilson TO, Podratz KC and Cliby WA: Aggressive surgical effort and improved survival in advanced-stage ovarian cancer. Obstet Gynecol 107: 77-85, 2006.

6. Kuhn W, Rutke S, Spathe K, Schmalfeldt B, Florack G, von Hundelshausen B, Pachyn D, Ulm K and Graeff H: Neoadjuvant chemotherapy followed by tumor debulking prolongs survival for patients with poor prognosis in International Federation of Gynecology and Obstetrics Stage IIIC ovarian carcinoma. Cancer 92: 2585-2591, 2001

7. Morrison J, Swanton A, Collins S and Kehoe S: Chemotherapy versus surgery for initial treatment in advanced ovarian epithelial cancer. Cochrane Database Syst Rev: CD005343, 2007.

8. Park TW and Kuhn WC: Neoadjuvant chemotherapy in ovarian cancer. Expert Rev Anticancer Ther 4: 639-647, 2004.

9. Bristow RE, Eisenhauer EL, Santillan A and Chi DS: Delaying the primary surgical effort for advanced ovarian cancer: A systematic review of neoadjuvant chemotherapy and interval cytoreduction. Gynecol Oncol 104: 480-490, 2007.

10. Goldie JH and Coldman AJ: A mathematic model for relating the drug sensitivity of tumors to their spontaneous mutation rate. Cancer Treat Rep 63: 1727-1733, 1979.

11. Norton L and Simon R: Tumor size, sensitivity to therapy, and design of treatment schedules. Cancer Treat Rep 61: 1307-1317, 1977.

12. Bristow RE and Chi DS: Platinum-based neoadjuvant chemotherapy and interval surgical cytoreduction for advanced ovarian cancer: a meta-analysis. Gynecol Oncol 10: 1070-1076, 2006.

13. Miller AB, Hoogstraten B, Staquet M and Winkler A: Reporting results of cancer treatment. Cancer 47: 207-214, 1981.
14. Kuhn W, Florack G and Roder J: The influence of upper abdominal surgery in perioperative morbidity and mortality in patients with advanced ovarian carcinoma FIGO III and FIGO IV. Int J Gynecol Cancer 8: 56-63, 1998.

15. A'Hern RP: Sample size tables for exact single-stage phase II designs. Stat Med 20: 859-866, 2001.

16. Zivanovic O, Eisenhauer EL, Zhou Q, Iasonos A, Sabbatini P, Sonoda Y, Abu-Rustum NR, Barakat RR and Chi DS: The impact of bulky upper abdominal disease cephalad to the greater omentum on surgical outcome for stage IIIC epithelial ovarian, fallopian tube, and primary peritoneal cancer. Gynecol Oncol 108: 287-292, 2008.

17. Everett EN, French AE, Stone RL, Pastore LM, Jazaeri AA, Andersen WA and Taylor PT Jr: Initial chemotherapy followed by surgical cytoreduction for the treatment of stage III/IV epithelial ovarian cancer. Am J Obstet Gynecol 195: 568-576, 2006.

18. Axtell AE, Lee MH, Bristow RE, Dowdy SC, Cliby WA, Raman S, Weaver JP, Gabbay M, Ngo M, Lentz S, et al: Multiinstitutional reciprocal validation study of computed tomography predictors of suboptimal primary cytoreduction in patients with advanced ovarian cancer. J Clin Oncol, 25: 384-389, 2007.

19. Brockbank EC, Ind TE, Barton DP, Shepherd JH, Gore ME, A'Hern R and Bridges JE: Preoperative predictors of suboptimal primary surgical cytoreduction in women with clinical evidence of advanced primary epithelial ovarian cancer. Int J Gynecol Cancer 14: 42-50, 2004.

20. Chi DS, Venkatraman ES, Masson V and Hoskins WJ: The ability of preoperative serum CA-125 to predict optimal primary tumor cytoreduction in stage III epithelial ovarian carcinoma. Gynecol Oncol 77: 227-231, 2000.

21. Gemer O, Lurian M, Gdalevich M, Kapustian V, Piura E, Schneider D, Lavie O, Levy T, Fishman A, Dgani R, et al: A multicenter study of CA 125 level as a predictor of non-optimal primary cytoreduction of advanced epithelial ovarian cancer. Eur J Surg Oncol 31: 1006-1010, 2005.

22. Fagotti A, Fanfani F, Ludovisi M, Lo Voi R, Bifulco G, Testa AC and Scambia G: Role of laparoscopy to assess the chance of optimal cytoreductive surgery in advanced ovarian cancer: a pilot study. Gynecol Oncol 96: 729-735, 2005.

23. Tate S, Hirai Y, Takeshima N and Hasumi K: CA125 regression during neoadjuvant chemotherapy as an independent prognostic factor for survival in patients with advanced ovarian serous adenocarcinoma. Gynecol Oncol 96: 143-149, 2005.

24. Rustin GJ: Use of CA-125 to assess response to new agents in ovarian cancer trials. J Clin Oncol 21 (Suppl 10): S187-S193, 2003.

25. Therasse P, Arbuck SG, Eisenhauer EA, Wanders J, Kaplan RS, Rubinstein L, Verweij J, van Glabbeke M, van Oosterom AT, Christian MC, et al: New guidelines to evaluate the response to treatment in solid tumors. European Organization for Research and Treatment of Cancer, National Cancer Institute of the United States, National Cancer Institute of Canada. J Natl Cancer Inst 92: 205-216, 2000.

26. Vasey PA: Role of docetaxel in the treatment of newly diagnosed advanced ovarian cancer. J Clin Oncol 21 (Suppl 10): S136-S144, 2003.

27. Vasey PA, Jayson GC, Gordon A, Gabra H, Coleman R, Atkinson R, Parkin D, Paul J, Hay A and Kaye SB: Phase III randomized trial of docetaxel-carboplatin versus paclitaxelcarboplatin as first-line chemotherapy for ovarian carcinoma. J Natl Cancer Inst 96: 1682-1691, 2004.

28. Crawford SC, Vasey PA, Paul J, Hay A, Davis JA and Kaye SB: Does aggressive surgery only benefit patients with less advanced ovarian cancer? Results from an international comparison within the SCOTROC-1 Trial. J Clin Oncol 23: 8802-8811, 2005.

29. Du Bois A, Weber B, Rochon J, Meier W, Goupil A, Olbricht S, Barats JC, Kuhn W, Orfeuvre H, Wagner U, et al: Addition of epirubicin as a third drug to carboplatin-paclitaxel in first-line treatment of advanced ovarian cancer: a prospectively randomized gynecologic cancer intergroup trial by the Arbeitsgemeinschaft Gynaekologische Onkologie Ovarian Cancer Study Group and the Groupe d'Investigateurs Nationaux pour l'Etude des Cancers Ovariens. J Clin Oncol 24: 1127-1135, 2006.

30. Ansquer Y, Leblanc E, Clough K, Morice P, Dauplat J, Mathevet P, Lhomme C, Scherer C, Tigaud JD, Benchaib M, et al: Neoadjuvant chemotherapy for unresectable ovarian carcinoma: a French multicenter study. Cancer 91: 2329-2334, 2001. 
31. Hou JY, Kelly MG, Yu H, McAlpine JN, Azodi M, Rutherford TJ and Schwartz PE: Neoadjuvant chemotherapy lessens surgical morbidity in advanced ovarian cancer and leads to improved survival in stage IV disease. Gynecol Oncol 105: 211-217, 2007.

32. Schwartz PE: Neoadjuvant chemotherapy for the management of ovarian cancer. Best Pract Res Clin Obstet Gynaecol 16: 585-596, 2002.

33. Vergote I, van Gorp T, Amant F, Neven P and Berteloot P: Neoadjuvant chemotherapy for ovarian cancer. Oncology 19: $1615-1630,2005$.
34. Vergote IB, Tropé CG, Amant F, Kristensen GB and Sardi JE: EORTC-GCG/NCIC-CTG Randomised Trial Comparing Primary Debulking Surgery With Neoadjuvant Chemotherapy in Stage IIIC-IV Ovarian, Fallopian Tube and Peritoneal Cancer (OVCA). 12th Biennial Meeting, Int Gynecol Cancer Soc (IGCS), 2008

35. Moher D, Schulz KF and Altman DG: The CONSORT statement: revised recommendations for improving the quality of reports of parallel-group randomised trials. Lancet 357: 1191-1194, 2005 